\title{
Pharmacologic Approach to Defective Protein Trafficking in the E637K-hERG Mutant with PD-118057 and Thapsigargin
}

\author{
Haiyan Mao ${ }^{19}$, Xiaoli $\mathrm{Lu}^{29}$, Justin Michael Karush ${ }^{39}$, Xiaoyan Huang ${ }^{1}$, Xi Yang ${ }^{1}$, Yanna Ba ${ }^{1}$, Ying Wang ${ }^{1}$, \\ Ningsheng Liư ${ }^{4}$, Jianqing Zhou ${ }^{1}$, Jiangfang Lian ${ }^{1 *}$
}

1 LiHuiLi Hospital, Medical School of NingBo University, NingBo, China, 2 People's Hospital of Anji County, HuZhou, China, 3 Department of Thoracic Surgery, Memorial Sloan-Kettering Cancer Center, New York, New York, United States of America, 4 Department of Pathology, Key Laboratory of Antibody Technique of Ministry of Health, Nanjing Medical University; Nanjing, China

\begin{abstract}
Background: Treatment of LQT2 is inadequate. Many drugs which can pharmacologically rescue defective protein trafficking in LQT2 also result in potent blockade of HERG current, negating their therapeutic benefit. It is reported that PD118057 and thapsigargin can rescue LQT2 without hERG channel blockade, but the precise mechanism of action is unknown. Furthermore, the effect of PD-118057 and thapsigargin on the dominant negative E637K-hERG mutant has not been previously investigated.

Objective: In this study, we investigated: (a) the effect of PD-118057 and thapsigargin on the current amplitudes of WThERG and WT/E637K-hERG channels; (b) the effect of PD-118057 and thapsigargin on the biophysical properties of WT-hERG and WT/E637K-hERG channels; (c) whether drug treatment can rescue channel processing and trafficking defects of the WT/ E637K-hERG mutant.

Methods: The whole-cell Patch-clamp technique was used to assess the effect of PD-118057 and thapsigargin on the electrophysiological characteristics of the rapidly activating delayed rectifier $\mathrm{K}^{+}$current $\left(\mathrm{I}_{\mathrm{kr}}\right)$ of the hERG protein channel. Western blot was done to investigate pharmacological rescue on hERG protein channel function.

Results: In our study, PD-118057 was shown to significantly enhance both the maximum current amplitude and tail current amplitude, but did not alter the gating and kinetic properties of the WT-hERG channel, with the exception of accelerating steady-state inactivation. Additionally, thapsigargin shows a similar result as PD-118057 for the WT-hERG channel, but with the exception of attenuating steady-state inactivation. However, for the WT/E637K-hERG channel, PD-118057 had no effect on either the current or on the gating and kinetic properties. Furthermore, thapsigargin treatment did not alter the current or the gating and kinetic properties of the WT/E637K-hERG channel, with the exception of opening at more positive voltages.
\end{abstract}

Conclusion: Our findings illustrate that neither PD-118057 nor thapsigargin play a role in correcting the dominant-negative effect of the E637K-hERG mutant.

Citation: Mao H, Lu X, Karush JM, Huang X, Yang X, et al. (2013) Pharmacologic Approach to Defective Protein Trafficking in the E637K-hERG Mutant with PD118057 and Thapsigargin. PLoS ONE 8(6): e65481. doi:10.1371/journal.pone.0065481

Editor: Andrea Cavalli, University of Bologna \& Italian Institute of Technology, Italy

Received February 3, 2013; Accepted April 26, 2013; Published June 19, 2013

Copyright: ( $) 2013$ Mao et al. This is an open-access article distributed under the terms of the Creative Commons Attribution License, which permits unrestricted use, distribution, and reproduction in any medium, provided the original author and source are credited.

Funding: The research was supported by the grants from: Advanced Key Scientific and Technological Programs of NingBo (2011C51001), Fund of NingBo Science and Technology Innovation team (2011B82015), National Natural Science Foundation of China (30772155), Zhejiang Provincial Program for the Cultivation of High-level Innovative Health talents, Ningbo personnel training project (first level), the project of Ningbo medicine and science(2009A02). The funders had no role in study design, data collection and analysis, decision to publish, or preparation of the manuscript.

Competing Interests: The authors have declared that no competing interests exist.

*E-mail: hjmpin@163.com

9 These authors contributed equally to this work.

\section{Introduction}

Mutation in the human ether-a-go-go-related gene (hERG or $\mathrm{KCNH} 2$ ) is responsible for reducing the rapidly activating component of the delayed rectifier potassium current $\left(\mathrm{I}_{\mathrm{Kr}}\right)$, which can prolong the QT interval and induce arrhythmia (Type 2 Long QT syndrome, LQT2) [1]. To date, genetic analyses have identified approximately 300 LQT2-associated KCNH2 muta- tions, which represent the most common mechanism of hERG channel dysfunction. Mutations in hERG lead to reduced expression of functional surface membrane channels as well as lower current magnitudes. Due to defective protein-trafficking, mutant channels are retained in the endoplasmic reticulum (ER) and fail to reach the plasma membrane $[2,3,4,5,6]$. It has been shown that correction of protein folding defects by pharmacologic chaperones (hERG channel blockers) can restore proper protein- 
trafficking [6]. However, their affinity for hERG channel blockade leads to acquired Long QT syndrome (LQTS) and severely limits their efficacy. This is due to binding of amino acid residues within the inner portion of the 6th transmembrane segment (S6) of hERG channel proteins $[7,8,9]$. Numerous types of drugs, with a wide range of chemical structures, are prone to hERG channel inhibition [10,11]. Many drugs have been removed from the market because of the potential lethality of hERG channel blockade, and screening for hERG block activity has become an important part of modern drug development [12]. As such, there is great interest in developing compounds that correct trafficking defects without hERG channel blockade.

Several drugs, classified as small molecule activators of hERG channels, have recently been reported which appear to rescue $\mathrm{I}_{\mathrm{Kr}}$ channels without significant hERG blockade, including RPR260243, NS1643, and PD-118057 (Table S1). Of these drugs, we tested PD-118057 because it has the most efficient current-enhancing effect on hERG current, without affecting the voltage dependence and kinetics of gating parameters, nor does it require open conformation of the hERG channel [13-15]. PD118057 represents one of the two major classes of hERG channel activators, but its precise mechanism of action is unknown, and safety and efficacy issues have yet to be adequately addressed. Unlike NS1643, whose activator effect is known to be potentiated by a mutated aromatic residue (Phe656) on S6 [14], no data exists regarding the effect of mutation on PD-118057.

Additionally, thapsigargin is a compound which inhibits endoplasmic reticulum Ca+-ATPase activity and has been shown to correct trafficking defects without hERG blockade [16]. We chose to assess thapsigargin because it promotes the relocation of intracellular proteins through its effect on $\mathrm{Ca}+$ dependent molecular chaperone activity, not through binding to the hERG channel itself. Thapsigargin, which is classified as a sesquiterpene lactone, can selectively rescue several different LQT2 mutations, including the $\mathrm{C}$ terminus mutation G601S and F805C, but not N470D [16]. The E637K-hERG mutant, which substitutes lysine for glutamic acid at position 637 in the pore-S6 loop transmembrane segment of hERG, has been identified as a dominantnegative mutation [14]. We undertook the present study to investigate: (a) the effect of PD-118057 and thapsigargin on the current amplitudes of WT-hERG and WT/E637K-hERG channels; (b) the effect of PD-118057 and thapsigargin on the biophysical properties of WT-hERG and WT/E637K-hERG channels; (c) whether drug treatment can rescue channel processing and trafficking defects of the WT/E637K-hERG mutant.

\section{Materials and Methods}

\section{Plasmid Construction}

HERG cDNA was subcloned into the pcDNA3 vector (Invitrogen,California, USA) at BamHI/EcoRI restriction sites. Mutant pcDNA3 E637K-hERG construct was made by overlap extension PCR and verified by DNA sequencing. The primer sequences were as follows: outer primers $5^{\prime}$-gccacgccagcaccggggccatgc-3' (forward) and 5'-gtgtggtcttgaacttcatggccaggge- $3^{\prime}$ (backward); mutagenesis primers $5^{\prime}$-caacaccaactcaaagaagatcttctcc- $3^{\prime}$ (forward) and 5'-ggagaagatcttctttgagttggtgttg-3' (backward). Plasmid DNA for mammalian expression was amplified in Escherichia coli TOP10 competent cells.

\section{Cell Lines and Drug Exposure}

Human embryonic kidney 293 (HEK293) cells were cultured in Dulbecco's Modified Eagle Medium (DMEM, Thermo scientific) supplemented with 10\% fetal bovine serum in 5\% CO2 incubator at $37^{\circ} \mathrm{C}$. HEK 293 cells were transiently transfected with $3.2 \mu \mathrm{g}$ of WT-hERG and/or $3.2 \mu \mathrm{g}$ of E637K-hERG plasmids using Lipofectamine $^{\mathrm{TM}} 2000$ according to the manufacturer's instruction (Invitrogen, California, USA). $0.8 \mu \mathrm{g}$ of pRK5-GFP plasmid was co-transfected to monitor transfection efficacy. Thapsigargin (Sigma, St. Louis, MO, USA; $1 \mathrm{mmol} / \mathrm{L}$ stock dissolved in DMSO), PD-118057 (Sigma, St. Louis, MO, USA; 5 mmol/L stock dissolved in DMSO) were added to the culture media for different time periods before analyzing. Final DMSO concentrations in medium was $<0.1 \%$.Incubating HEK 293 cells expressing WT-hERG, WT/E637K-hERG or E637K-hERG overnight in $0.1 \%$ DMSO had no effect on $\mathrm{I}_{\mathrm{hERG}}$ or complex glycosylation.

\section{Whole-cell Patch-Clamp Recordings}

To examine the effect of drug treatment on WT/E637K-hERG by patch clamp technique, HEK293 cells were harvested at 24 (DNA plasmid only) or 48 hours (DNA plasmid followed by drugs) and superfused with 4-(2-hydroxyethyl)-1-piperazine ethane sulphonic acid (HEPES)-buffered Tyrode solution containing $137 \mathrm{mM} \mathrm{NaCl}, 4 \mathrm{mM} \mathrm{KCl}, 1.8 \mathrm{mM} \mathrm{CaCl}{ }_{2}, 1 \mathrm{mM} \mathrm{MgCl}_{2}$, $10 \mathrm{mM}$ glucose, and $10 \mathrm{mM}$ HEPES (pH 7.4 adjusted with $\mathrm{NaOH}$ ). The internal pipette solution contained $130 \mathrm{mM} \mathrm{KCl}$, $1 \mathrm{mM} \mathrm{MgCl}_{2}, 5 \mathrm{mM}$ [Ethylenebis(oxyethlenenitrilo)]-Tetraacetic

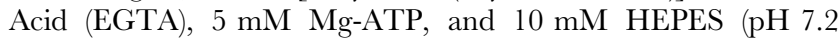
adjusted with $\mathrm{KOH})$ as previously described $[15,16,17,18]$. Membrane currents were recorded in whole-cell configuration using pipettes with a tip resistance of 2 to $5 \mathrm{Mohms}$ when filled with the internal solution. The electrodes were connected to an Axopatch 700A amplifier (Axon Instruments, California, USA) and digitized at $2 \mathrm{kHz}$ with an analogue-to-digital converter (DigiData 1200B; Axon Instruments, California, USA). All experiments were done at room temperature.

\section{Western Blot Analysis}

Western blot analysis of hERG protein was performed as previously described [19]. Briefly, HEK293 cells were solubilized in ice-cold Radio-Immunoprecipitation Assay (RIPA) buffer with freshly added protease inhibitors and phenylmethanesulfony fluoride (PMSF) (Solarbio,Beijing,China). Proteins were separated on $8 \%$ SDS-polyacrylamide gels and transferred to polyvinylidene difluoride (PVDF) membranes. Membranes were blocked for 2-hrs with blocking solution $(5 \%$ nonfat dry milk powder and $0.1 \%$ Tween -20 in TBS) and subsequently incubated with rabbit polyclonal anti-hERG antibody (Alomone Labs,Jerusalem, Israel) at $4{ }^{\circ} \mathrm{C}$ over night, followed by alkaline phosphatase goat antirabbit IgG (ZSGB-BIO, Beijing, China) for 2-hrs at room temperature. Protein bands were detected with the alkaline phosphatase substrate BCIP/NBT colorimetric determination kit (ZSGB-BIO, Beijing, China).

\section{Statistical Analysis}

The pCLAMP Ver. 9.2 software (Axon Instruments, California, USA) was used to generate voltage clamp protocols, acquire data and analyze current traces. Voltage-dependence activation was determined by fitting the peak tail current $\left(\mathrm{I}_{\text {tail }}\right)$ with Boltzmann function: $\mathrm{I} / \mathrm{Imax}=1-\left\{\left(1+\exp \left[\left(\mathrm{Vt}-\mathrm{V}_{1 / 2}\right) / \mathrm{k}\right]\right\}^{-1} \cdot \mathrm{V}_{1 / 2}\right.$ represents the half-maximal voltage and $\mathrm{k}$ is the slope factor. Time constants of inactivation or recovery from inactivation were fitted by single exponential standard. Fast and slow time constants of deactivation were fitted by double exponential standard. Results were expressed as means $\pm \mathrm{SE}$, and statistical analyses were performed with Student's t-test. Results were considered to be statistically significant when $P<0.05$. 

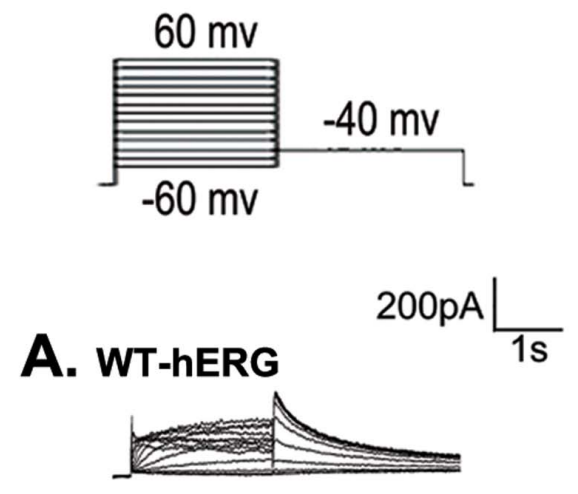

B. WT-hERG and PD-118057

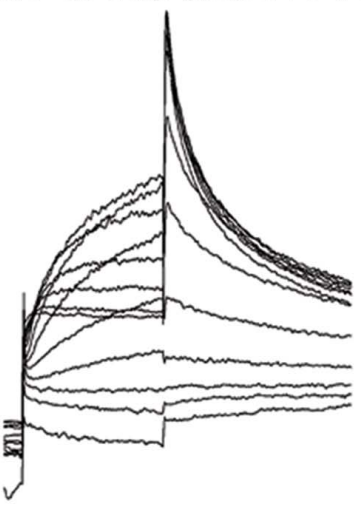

C. WT/E637K-hERG

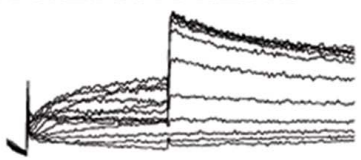

D. WT/E637K-hERG and PD-118057

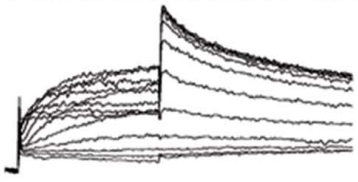

E. E637K-hERG

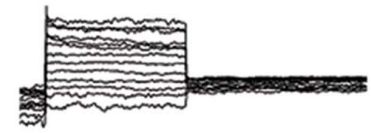

F. E637K-hERG and PD-118057

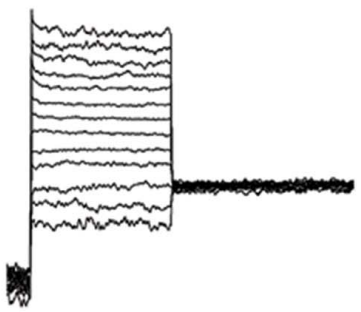

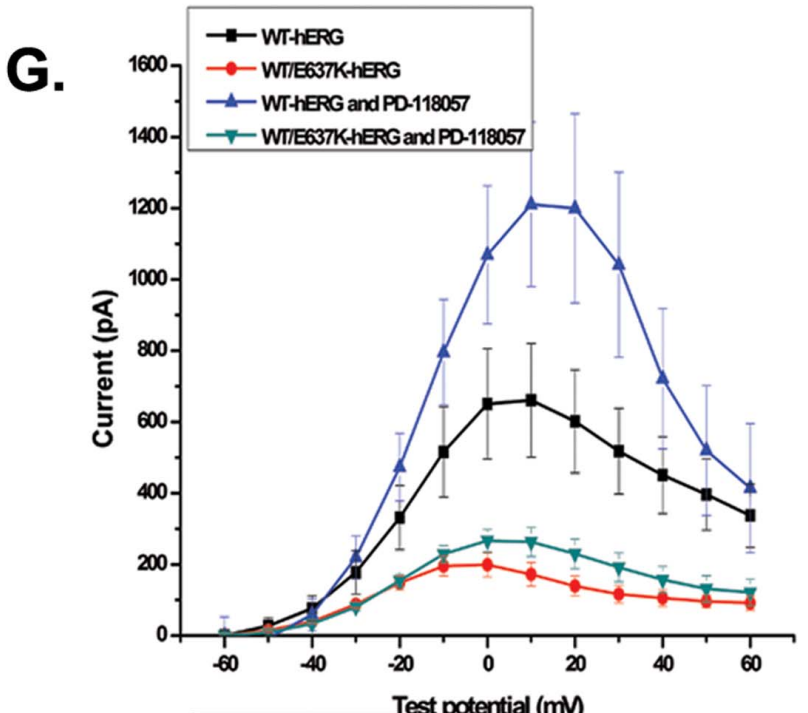

H.

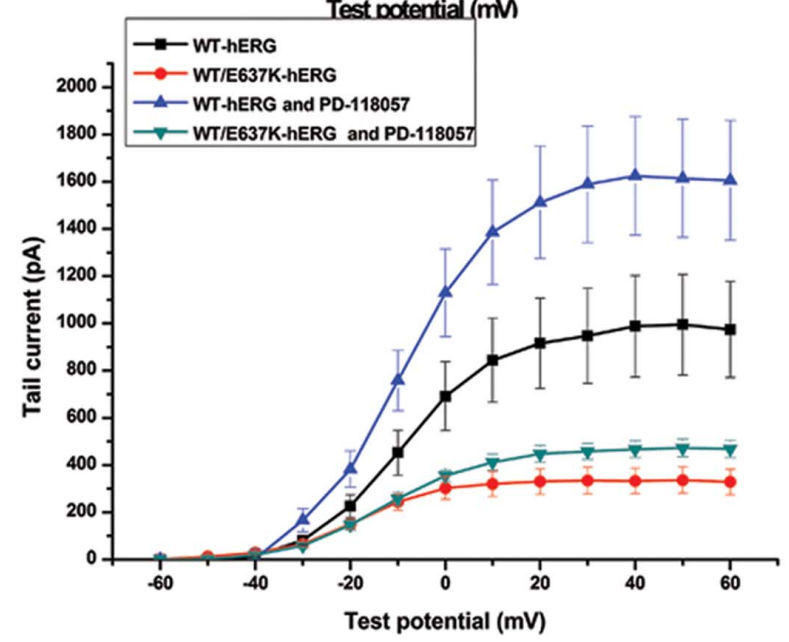

I.

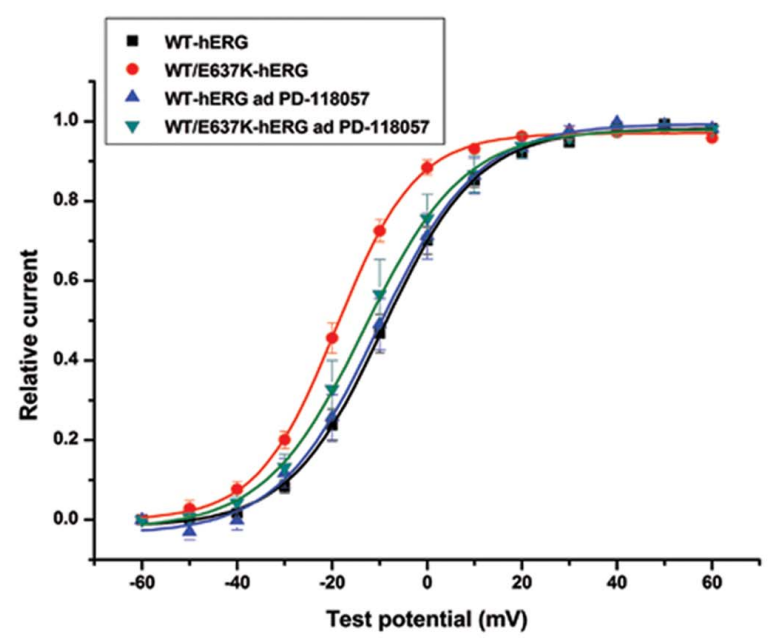

Figure 1. Effect of PD-118057 ( $3 \mu \mathbf{M})$ on voltage-dependent activation of hERG channel. Inset shows the voltage clamp protocol. a-f: Representative current traces in HEK293 cells transfected with WT-hERG, WT/E637K-hERG, and E637K-hERG in the presence or absence of PD-118057. $\mathrm{g}, \mathrm{h}$ : Current-voltage (I-V) relationships for peak and tail current amplitudes of WT-hERG and WT/E637K-hERG transfected cells in the presence and absence of PD-118057. i: Amplitudes of tail currents of WT-hERG and WT/E637K-hERG channels in the presence or absence of PD-119057 are plotted as a function of the test potential and fitted to a Boltzmann function $(n=6)$.

doi:10.1371/journal.pone.0065481.g001 

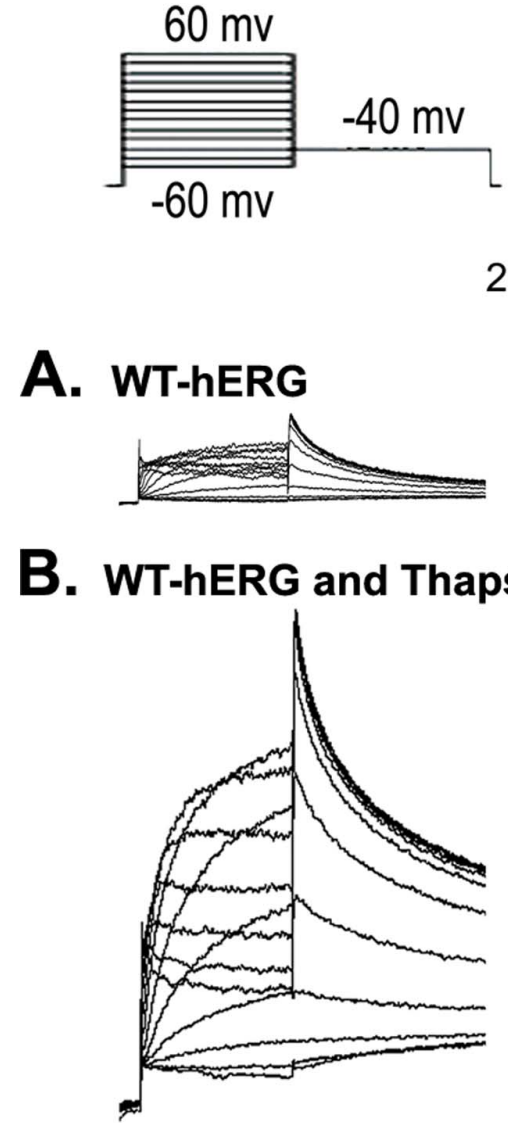

C. WT/E637K-hERG

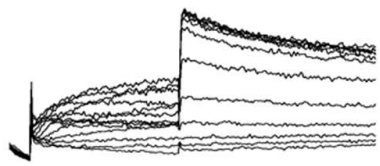

D. WT/E637K-hERG and Thapsigargin

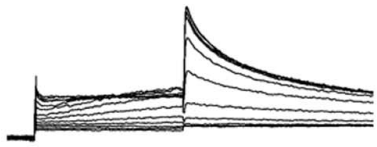

E. E637K-hERG

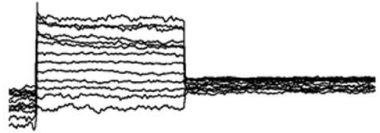

F. E637K-hERG and Thapsigargin

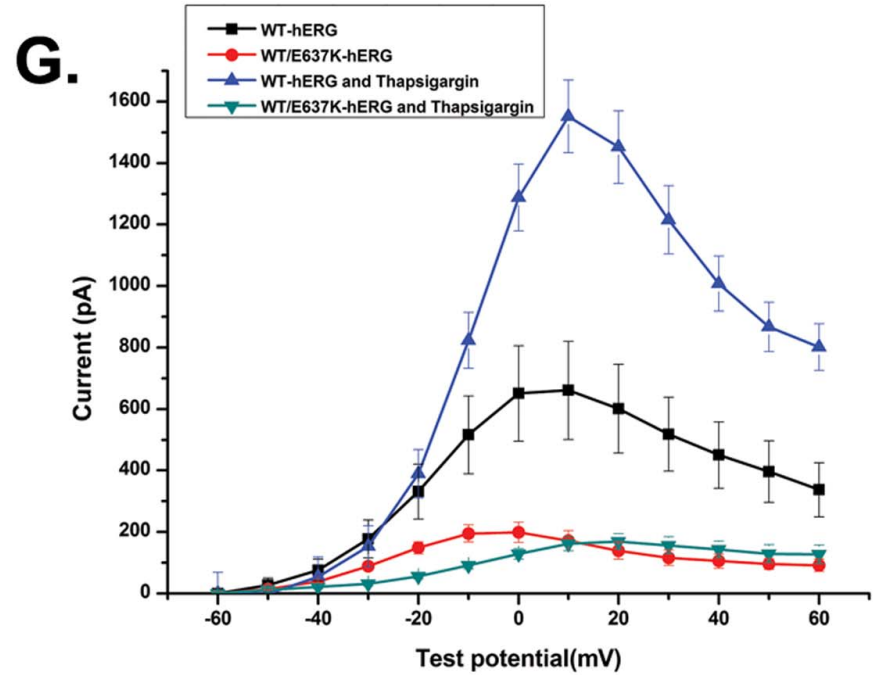

H.
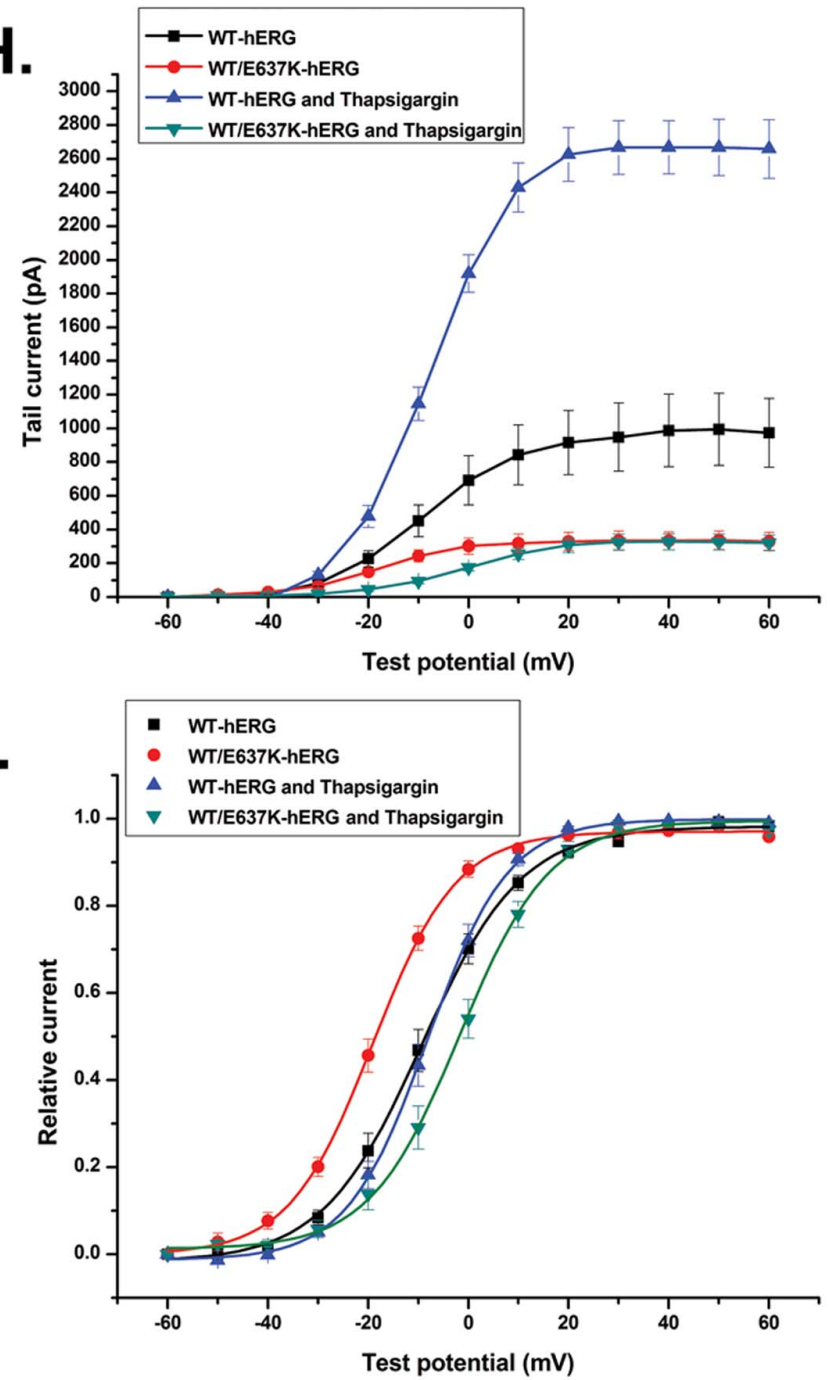

Figure 2. Effect of thapsigargin $(1 \mu \mathrm{M})$ on voltage-dependent activation of hERG channel. Inset shows the voltage clamp protocol. a-f: Representative current traces in HEK293 cells transfected with WT-hERG, WT/E637K-hERG, and E637K-hERG in the presence or absence of thapsigargin. $\mathrm{g}, \mathrm{h}$ : Current-voltage (I-V) relationships for peak and tail current amplitudes of WT-hERG and WT/E637K-hERG transfected cells in the presence or absence of thapsigargin. i: Amplitudes of tail currents of WT-hERG or WT/E637K-hERG channels in the presence or absence of thapsigargin are plotted as a function of the test potential and fitted to a Boltzmann function $(n=6)$.

doi:10.1371/journal.pone.0065481.g002 
A. WT-hERG

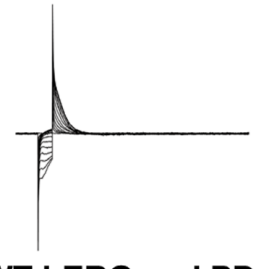

B. WT-hERG and PD-118057

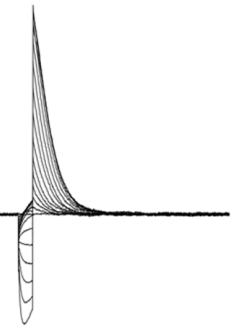

C. WT/E637K-hERG

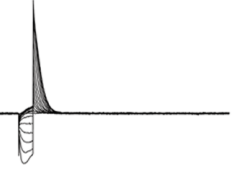

D. WT/E637K-hERG and PD-118057

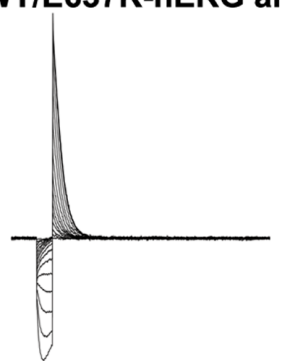

F. WT-hERG<smiles>CCCCC(C)C</smiles>

G. WT-hERG and Thapsigargin

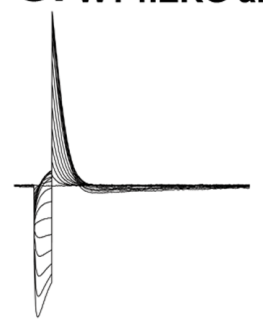

H. WT/E637K-hERG

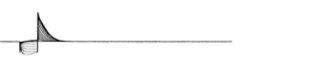

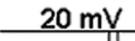

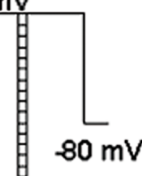

$-130 \mathrm{mV}$

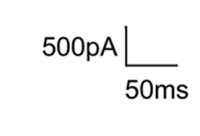

$50 \mathrm{~ms}$ 


\section{A. WT-hERG}
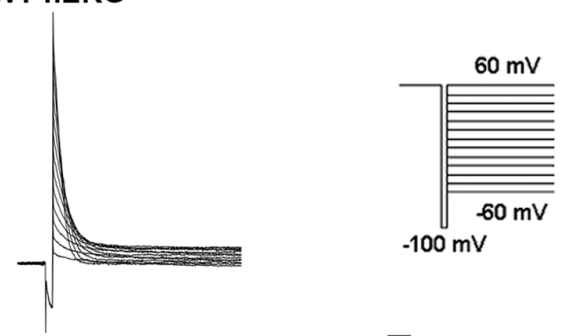

1500pA

$50 \mathrm{~ms}$

B. WT-hERG and PD-118057

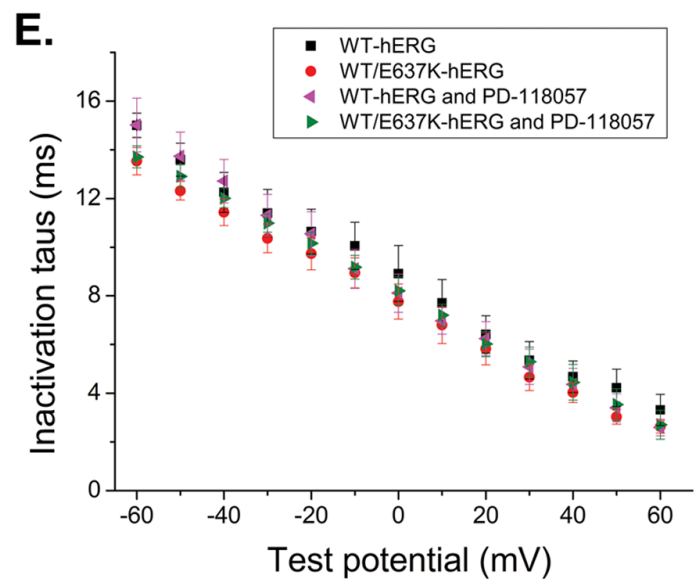

D. WT/E637K-hERG and PD-118057
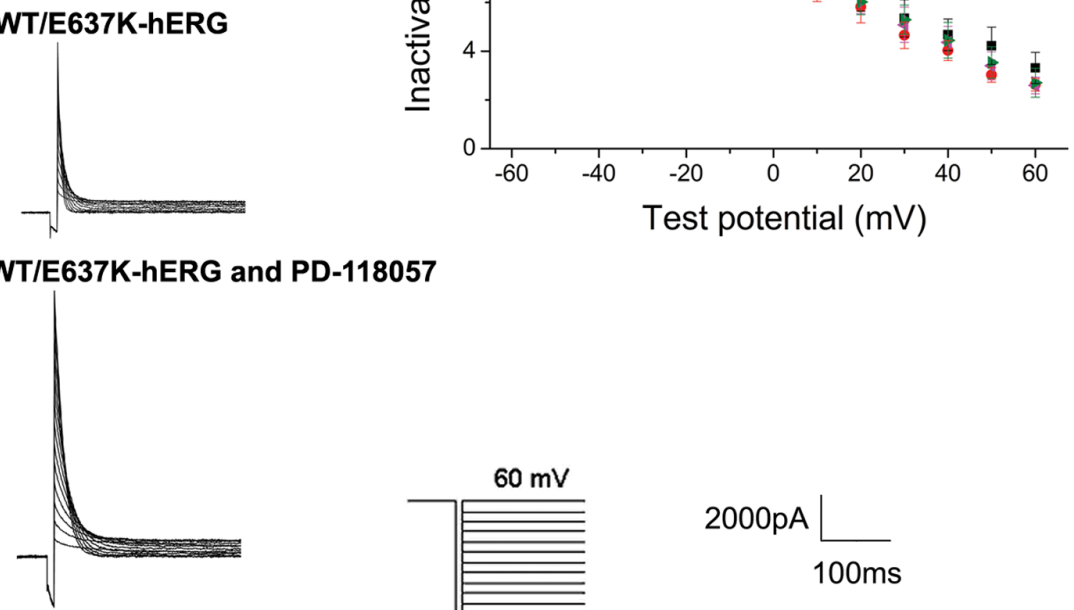

F. WT-hERG
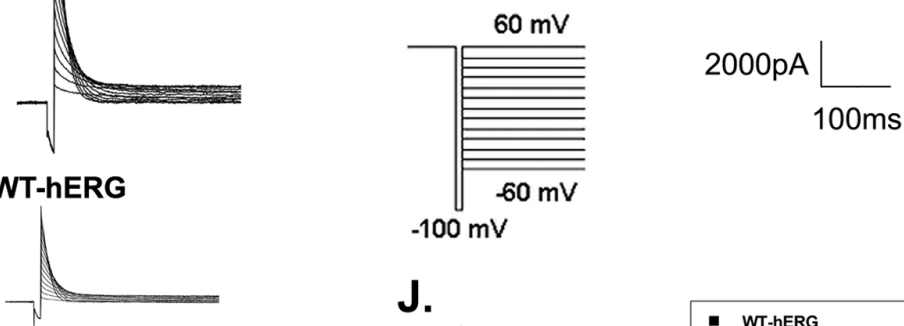

G. WT-hERG and Thapsigargin

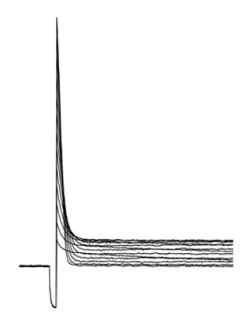

\section{H. WT/E637K-hERG}

\section{J.}
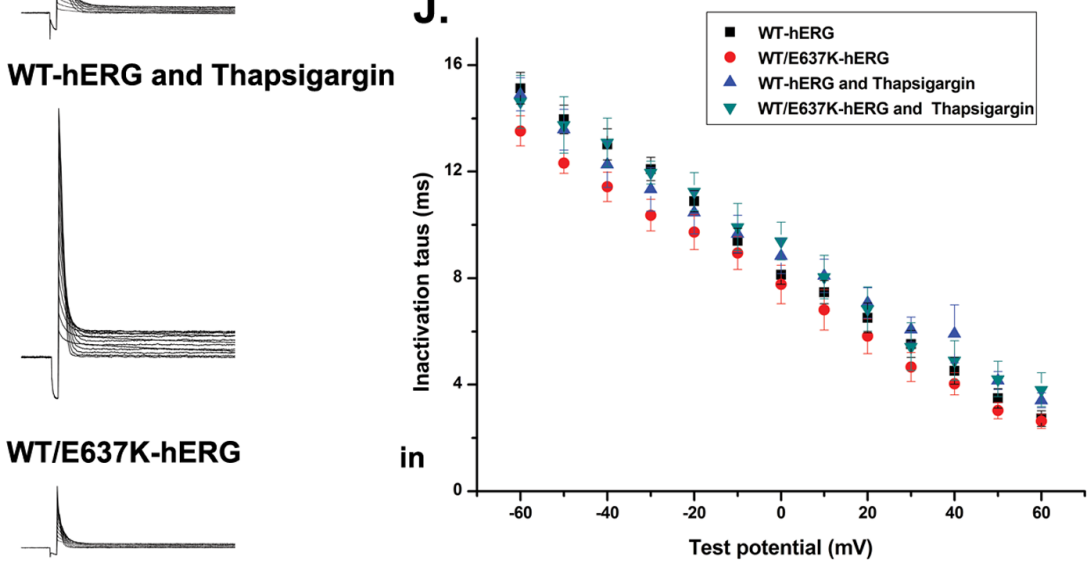

I. WT/E637K-hERG and Thapsigargin

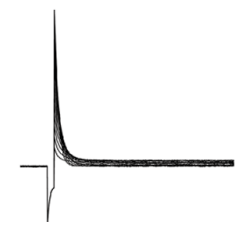


Figure 4. Effect of PD-118057 (3 $\mu \mathrm{M})$ and thapsigargin $(1 \mu \mathrm{M})$ on time courses of inactivation of hERG channel. Inset shows the voltage clamp protocol. a-d: Representative current traces of time courses and inactivation time constants $($ tau, $\tau$ ) in HEK293 cells transfected with WT-hERG or WT-hERG/E637K-hERG plasmids in the presence or absence of PD-118057 and thapsigargin. e: $\tau$ was measured by fitting inactivation currents of WT-hERG or WT-hERG/E637K-hERG channel, in the presence or absence of PD-118057 and thapsigargin during test pulse at each potential, with a single exponential function $(n=6)$.

doi:10.1371/journal.pone.0065481.g004

\section{Results}

Effect of PD-118057 and Thapsigargin on WT-hERG and WT/E637K-hERG Current Amplitudes

We first measured the peak and tail currents in untreated HEK293 cells expressing WT-hERG or WT/E637K-hERG channels. Currents were elicited by voltage clamp protocol as previously described [20,21]. Cells were depolarized to test voltages between $-60 \mathrm{mV}$ and $60 \mathrm{mV}$ in $10 \mathrm{mV}$ increments for $2 \mathrm{~s}$ from a $-80 \mathrm{mV}$ holding potential, followed by $-40 \mathrm{mV}$ for $4 \mathrm{~s}$ to elicit tail currents. Figure 1 shows representative whole cell currents and the corresponding current-voltage $(\mathrm{I}-\mathrm{V})$ relationship of the maximal and tail current amplitudes. As expected, untreated WT/E637K-hERG cells demonstrated a significant reduction in maximal current amplitude compared to WT-hERG cells $(198.66 \pm 33.39 \mathrm{pA}$ versus $660.67 \pm 159.55 \mathrm{pA} ; \mathrm{n}=6, \mathrm{p}<0.05$, Fig. $1 \mathrm{G}$ and $2 \mathrm{G}$ ). A similar reduction in tail current was observed in WT/E637K-hERG expressing cells compared to WT-hERG (333.12 $\pm 1.48 \mathrm{pA}$ versus $981.28 \pm 5.94 \mathrm{pA} ; \mathrm{n}=6, \mathrm{P}<0.05$, Fig. $1 \mathrm{H}$ and $2 \mathrm{H}$ ), confirming the negative effect of WT/E637K-hERG expression.

Next, we treated HEK293 cells expressing WT-hERG or WT/ E637K-hERG channels with either PD-118057 $(3 \mu \mathrm{M})$ or thapsigargin $(1 \mu \mathrm{M})$ and compared current amplitudes to untreated controls. Single drug concentrations were chosen due to previous optimization of these drugs in terms of both efficacy of hERG current rescue as well as prevention of pharmacologically induced QT prolongation [13,17] In WT-hERG expressing cells, PD-1 18057 treatment resulted in a significant increase in both maximal current amplitude $(1310.96 \pm 230.65 \mathrm{pA}$ versus $660.67 \pm 159.55 \mathrm{pA}$ control; $\mathrm{n}=6, \mathrm{p}<0.05$, Fig. $1 \mathrm{G})$ and tail current amplitude $(1620.20 \pm 11.12 \mathrm{pA}$ versus $981.28 \pm 5.94 \mathrm{pA}$ control; $\mathrm{n}=6, \mathrm{P}<0.05$, Fig. $1 \mathrm{H}$ ), but failed to rescue either the maximal current amplitude $(279.47 \pm 29.05 \mathrm{pA}$ versus $198.66 \pm 33.39 \mathrm{pA}$ control; $\mathrm{n}=6, \mathrm{p}>0.05$, Fig. 1G) or the tail current amplitude $(468.43 \pm 2.06 \mathrm{pA}$ versus $333.12 \pm 1.48 \mathrm{pA}$ control; $\mathrm{n}=6, \mathrm{p}>0.05)$ in cells expressing WT/E637K-hERG. Similarly, thapsigargin treatment also enhanced both the maximal current amplitude $(1551.79 \pm 117.55 \mathrm{pA}$ versus $660.67 \pm 159.55 \mathrm{pA}$ control; $\mathrm{n}=6, \mathrm{p}<0.05$, Fig. $2 \mathrm{G}$ ) and tail current amplitude $(2680.42 \pm 10.65 \mathrm{pA}$ versus 981.28 $\pm 5.94 \mathrm{pA}$ control; $\mathrm{n}=6$, $\mathrm{p}<0.05$, Fig. 2H) in WT-hERG expressing cells but failed to rescue either the maximal current amplitude $(169.18 \pm 25.00 \mathrm{pA}$ versus $198.66 \pm 33.39 \mathrm{pA}$ control; $\mathrm{n}=6, \mathrm{p}>0.05$, Fig. $2 \mathrm{G}$ ) or the tail current amplitude $(329.84 \pm 2.63 \mathrm{pA}$ versus $333.12 \pm 1.48 \mathrm{pA}$ control; $\mathrm{n}=6, \mathrm{p}>0.05$, Fig. $2 \mathrm{H})$ in WT/E637K-hERG cells.

\section{Effect of PD-118057 and Thapsigargin on Gating Properties of WT-hERG and WT/E637K-hERG Protein Channels}

To assess the effect of PD-118057 and thapsigargin on the voltage dependence of protein channel activation, we normalized the tail currents of cells expressing WT-hERG and WT/E637KhERG before and after drug treatment. Currents were plotted as a function of the test potential and fitted to a Boltzmann function, where $\mathrm{V}_{1 / 2}$ is the half-maximum activation voltage and $\mathrm{k}$ is the slope factor representing the steepness of the voltage dependence.
In cells expressing WT-hERG, PD-1 18057 treatment resulted in no significant change in either the activation voltage $\left(V_{1 /}\right.$ ${ }_{2}=-10.38 \pm 0.54 \mathrm{mV}$ versus $-8.66 \pm 0.31 \mathrm{mV}$ control; $\mathrm{n}=6$, $\mathrm{p}>0.05$; fig. 1I) or the slope factor $(\mathrm{k}=10.49 \pm 0.31 \mathrm{mV}$ versus $9.99 \pm 0.34 \mathrm{mV}$ control; $\mathrm{n}=6, \mathrm{p}>0.05$; fig. 1I), which is consistent with the observations by Zhou and colleagues [15]. Similarly, thapsigargin did not alter the activation voltage $\left(\mathrm{V}_{1}\right.$ / ${ }_{2}=-7.90 \pm 0.23 \mathrm{mV}$ versus $-8.66 \pm 0.31 \mathrm{mV}$ control; $\mathrm{n}=6$, $\mathrm{p}>0.05$; fig. 2I) or slope factor $(\mathrm{k}=8.06 \pm 0.20$ versus $9.99 \pm 0.43 \mathrm{mV}$ control; $\mathrm{n}=6, \mathrm{p}>0.05$; fig. 2I) in WT-hERG cells. In cells expressing WT/E637K-hERG, PD-118057 treatment also resulted in no difference in activation voltage $\left(V_{1}\right.$ / ${ }_{2}=-13.26 \pm 0.34 \mathrm{mV}$ versus $-18.24 \pm 0.30 \mathrm{mV}$ control; $\mathrm{n}=6$, $\mathrm{p}>0.05$; fig. 1I) or slope factor $(\mathrm{k}=10.45 \pm 0.27 \mathrm{mV}$ versus 8.34 $\pm 0.27 \mathrm{mV}$ control; $\mathrm{n}=6, \mathrm{p}>0.05$; fig. 1I) However, thapsigargin treatment did result in a significant change in activation voltage in WT/E637K-hERG expressing cells $\left(\mathrm{V}_{1}\right.$ / ${ }_{2}=-1.20 \pm 0.44 \mathrm{mV}$ versus $-18.24 \pm 0.30 \mathrm{mV}$ control; $\mathrm{n}=6$, $\mathrm{p}<0.05$, Fig. 2I) but no change in slope factor $(\mathrm{k}=8.98 \pm 0.39 \mathrm{mV}$ versus $8.34 \pm 0.27 \mathrm{mV}$ control). These results indicate that WT/E637K-hERG channels open at more positive voltages after thapsigargin treatment.

Steady-state inactivation. To analyze the steady-state inactivation, test potentials between $-130 \mathrm{mV}$ and $20 \mathrm{mV}$ in $10 \mathrm{mV}$ increments for $20 \mathrm{~ms}$ were applied after a depolarizing pulse back to $20 \mathrm{mV}$ for $4 \mathrm{~s}$. This was followed by a test pulse to $20 \mathrm{mV}$ for $500 \mathrm{~ms}$ before returning to a holding potential of $-80 \mathrm{mV}$ (Fig. 3, inset). Figures $3 \mathrm{~A}-\mathrm{D}$ and $3 \mathrm{~F}-\mathrm{I}$ show the representative voltage clamp recordings and figure $3 \mathrm{E}$ and $3 \mathrm{~J}$ depict the normalized steady-state inactivation curves. In WThERG cells, PD-118057 treatment resulted in a significant change in steady-state inactivation $\left(\mathrm{V}_{1 / 2}=-55.83 \pm 1.42 \mathrm{mV}\right.$ versus $-39.07 \pm 3.13 \mathrm{mV}$ control, $\mathrm{n}=6, \mathrm{p}<0.05)$ but no change in slope factor $(\mathrm{k}=20.20 \pm 1.52 \mathrm{mV}$ versus $14.94 \pm 2.88 \mathrm{mV}$ control). Thapsigargin treatment also resulted in an alteration in steadystate inactivation $\left(\mathrm{V}_{1 / 2}=-28.43 \pm 4.85 \mathrm{mV}\right.$ versus $-39.07 \pm 3.13 \mathrm{mV}$ control, $\mathrm{n}=6, \mathrm{p}<0.05)$ in WT-hERG cells but no change in slope factor $(\mathrm{k}=12.19 \pm 4.21 \mathrm{mV}$ versus $14.94 \pm 2.88 \mathrm{mV}$ control). WT/E637K-hERG channels were largely unaffected by either PD-118057 $\left(\mathrm{V}_{1}\right.$ / ${ }_{2}=-58.69 \pm 1.77 \mathrm{mV}, \mathrm{k}=16.64 \pm 2.13 \mathrm{mV}$ ) or thapsigargin treatment $\left(\mathrm{V}_{1 / 2}=-62.62 \pm 2.15 \mathrm{mV}, \mathrm{k}=19.99 \pm 2.23 \mathrm{mV}\right)$ compared to untreated control $\left(\mathrm{V}_{1 / 2}=-53.40 \pm 2.53 \mathrm{mV}\right.$, $\mathrm{k}=15.66 \pm 2.52 \mathrm{mV}, \mathrm{n}=6, \mathrm{p}>0.05)$. Our results demonstrate that both PD-1 18057 and thapsigargin treatment alter the steady-state inactivation kinetics of WT-hERG channels, but fail to affect WT/ E637K-hERG channels.

Inactivation time course. We next estimated the inactivation time course as previously described [18]. First, cells were depolarized from a holding potential of $-80 \mathrm{mV}$ to $60 \mathrm{mV}$ for $2 \mathrm{~s}$, hyperpolarized to $-100 \mathrm{mV}$ for $10 \mathrm{~ms}$ to allow hERG protein channels to recover from inactivation, and then depolarized to a test potential between $-60 \mathrm{mV}$ and $60 \mathrm{mV}$ in $10 \mathrm{mV}$ increments for $3 \mathrm{~s}$ to record the inactivation current (Fig. 4, inset). Figure 4A$\mathrm{D}$ and $4 \mathrm{~F}-\mathrm{I}$ depict the typical current tracings of cells expressing WT-hERG and WT/E637K-hERG in the presence and absence of PD-118057 or thapsigargin. The time constants of inactivation 
A. WT-hERG

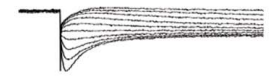

B. WT-hERG and PD-11805

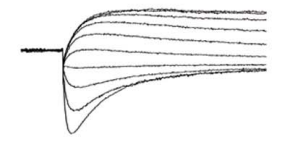

C. WT/E637K-hERG

D. WT/E637K-hERG and PD7

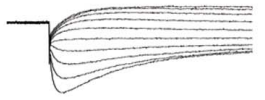

F. WT-hERG

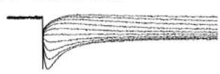

G. WT-hERG and Thapsigargin

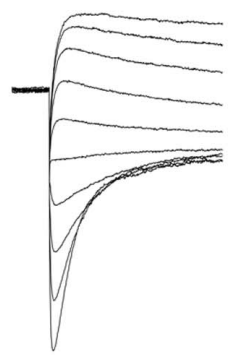

\section{H. WT/E637K-hERG}

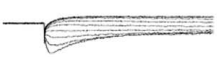

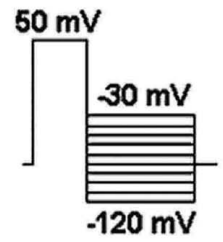

E.

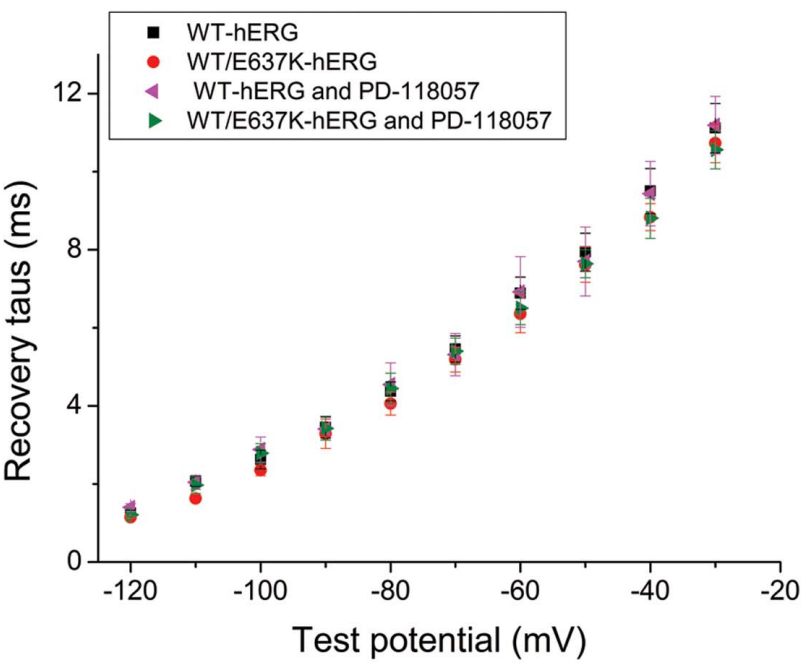

1000pA $\frac{}{100 \mathrm{~ms}}$

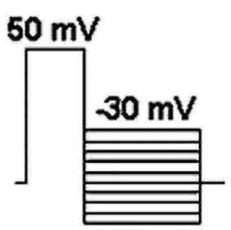

$-120 \mathrm{mV}$

J.

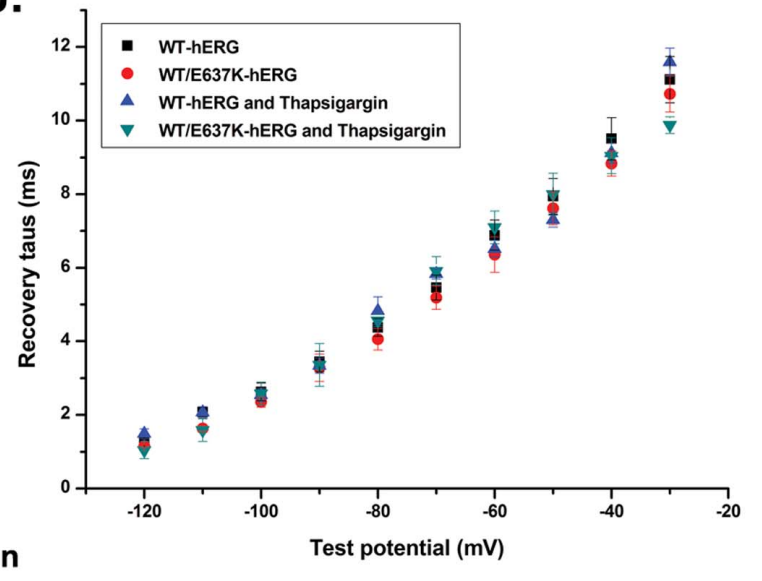

\section{WT/E637K-hERG and Thapsigargin}

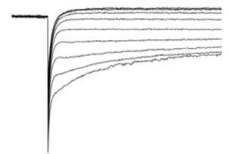

Figure 5. Effect of PD-118057 (3 $\mu \mathrm{M})$ and thapsigargin (1 $\mu \mathrm{M})$ on recovery from inactivation of hERG channel. Insert shows the voltage clamp protocol. a-d: Representative recovery from inactivation traces in HEK293 cells transfected with WT-hERG or WT/E637K-hERG plasmids in the presence or absence of PD-118057 and thapsigargin. e: Time constants (tau, $\tau$ ) for hERG channel recovery from inactivation are plotted as a function of test voltages for WT-hERG or WT/E637K-hERG plasmids in the presence or absence of drug $(n=6)$. doi:10.1371/journal.pone.0065481.g005 
A. WT-hERG

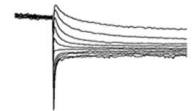

B. WT-hERG and PD-118057

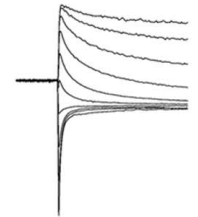

C. WT/E637K-hERG<smiles>C#CCCC</smiles>

D. WT/E637K-hERG and PD-118057

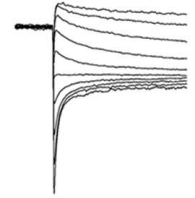

F. WT-hERG

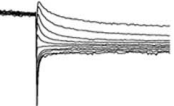

\section{G. WT-hERG and Thapsigargin}

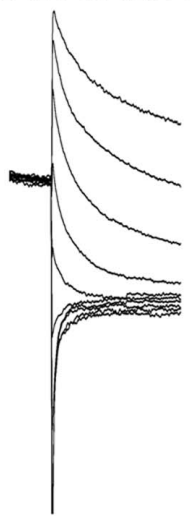

H. WT/E637K-hERG

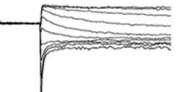

$500 \mathrm{pA}$

$1000 \mathrm{~ms}$

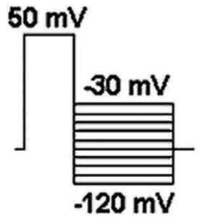

E.

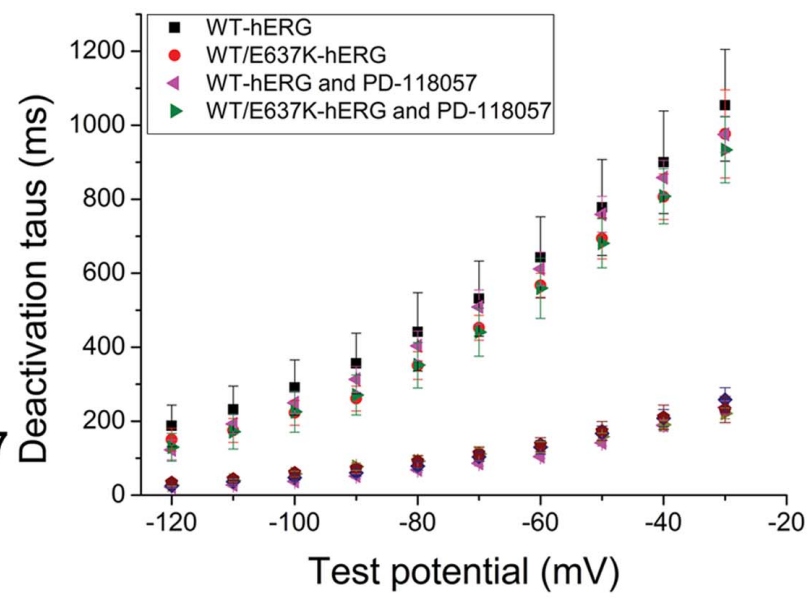

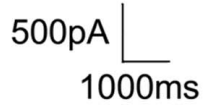

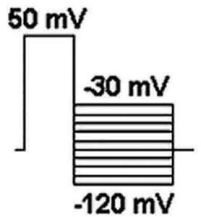

J.

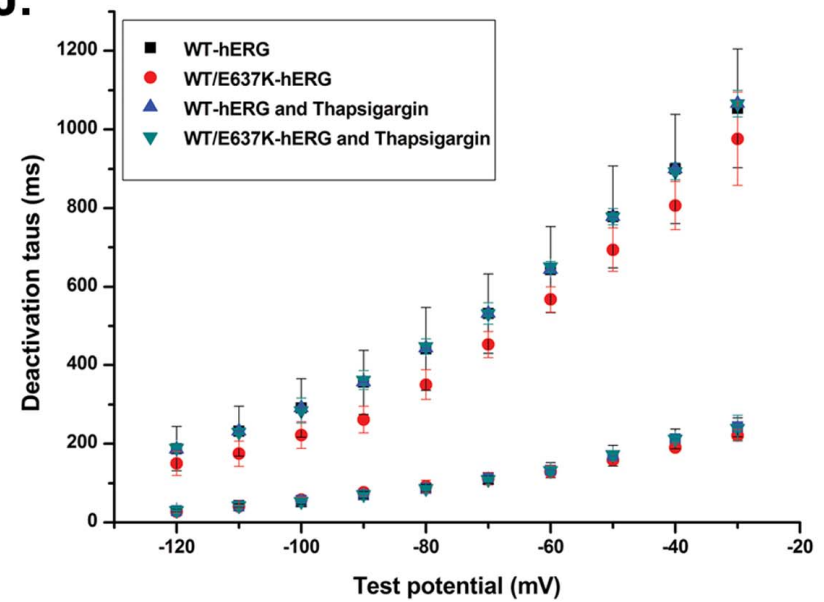

I. WT/E637K-hERG and Thapsigargin

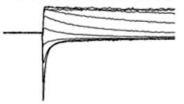

Figure 6. Effect of PD-118057 (3 $\mu \mathrm{M})$ and thapsigargin (1 $\mu \mathrm{M})$ on deactivation of hERG channel. Insert shows the voltage clamp protocol. a-d: Representative deactivation traces in HEK293 cells transfected with WT-hERG or WT/E637K-hERG in the presence or absence of PD-118057 and thapsigargin (arrow marks the deactivation phase). e: Fast and slow components of deactivation time constants (tau, $\tau$ ) are plotted as a function of test potentials for WT-hERG or WT/E637K-hERG plasmids in the presence or absence of drug $(n=6)$.

doi:10.1371/journal.pone.0065481.g006 


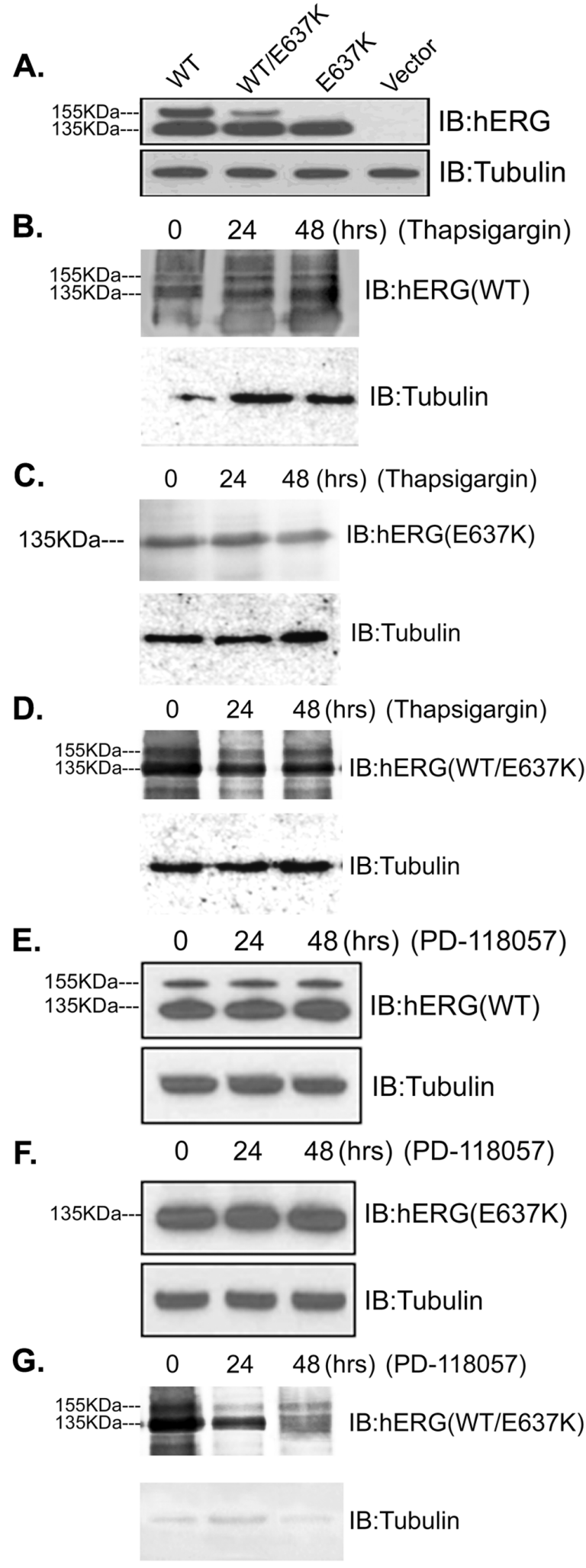

Figure 7. Analysis of hERG protein expression in HEK293 cells. a. Representative protein expression of untreated WT-hERG, WT/E637KhERG, and E637K-hERG channels. b-d. Representative protein expression of WT-hERG, WT/E637K-hERG and E637K-hERG channels treated with $1 \mathrm{uM}$ thapsigargin for $24 \mathrm{~h}$ to $48 \mathrm{~h}$, respectively. Thapsigargin has no effect on the protein expression profile of either WT/E637K-hERG or E637K-hERG. e-f. Representative protein expression of WT-hERG, WT/
E637K-hERG and E637K-hERG channels treated with $3 \mu \mathrm{M}$ PD-118057 for $24 \mathrm{~h}$ to $48 \mathrm{~h}$, respectively. As demonstrated, PD-118057 also has no effect on the protein expression profile of either WT/E637K-hERG or E637K-hERG channels.

doi:10.1371/journal.pone.0065481.g007

were fitted as a single exponential function with the average $( \pm \mathrm{SE})$ plotted in figure 4E and 4J. We found that exposure to PD-1 18057 or thapsigargin had no significant effect on the inactivation time course of either WT-hERG or WT/E637K-hERG channels $(\mathrm{n}=6, \mathrm{P}>0.05)$. These results demonstrate that neither PD118057 nor thapsigargin affects the inactivation kinetics of WThERG or WT/E637K-hERG channels.

Recovery from inactivation. To analyze the recovery from inactivation, cells were depolarized to $50 \mathrm{mV}$ for $1.5 \mathrm{~s}$ and then repolarized to a test potential between $-120 \mathrm{mV}$ and $-30 \mathrm{mV}$ for $3 \mathrm{~s}$ to elicit tail currents (Fig. 5, inset). Figure 5A-D and 5F-I show the current tracings from cells expressing WT-hERG and WT/ E637K-hERG channels in the presence and absence of PD118057 or thapsigargin. Recovery from inactivation is represented by the initial phase of these currents, which were fitted as a single exponential function of the test potential to obtain the time constant of recovery from inactivation (Fig. 5E and 5J). As our data shows, the inactivation properties of cells expressing either WT-hERG or WT/E637K-hERG channels were not affected by PD-1 18057 or thapsigargin $(\mathrm{n}=6, \mathrm{P}>0.05)$.

Deactivation. Deactivation was measured by using the same protocol as recovery from inactivation (Fig. 6, inset). Figures 6A-D and $6 \mathrm{~F}-\mathrm{I}$ depict the typical current tracings from cells expressing WT-hERG and WT/E637K-hERG before and after exposure to PD-118057 or thapsigargin. The fast and slow deactivation time courses were measured by fitting deactivating tail currents during test pulses to a double exponential function. As the results show (Fig. 6E and 6J), no significant differences were found in the fast and slow time constants of deactivation for WT-hERG and WT/ E637K-hERG channels treated by PD-118057 or thapsigargin compared to control $(\mathrm{n}=6, \mathrm{P}>0.05)$.

\section{Effect of PD-118057 and Thapsigargin on Channel Processing and Trafficking}

To study whether PD-118057 and thapsigargin can pharmacologically rescue the dominant-negative effect of E637K-hERG channel function, hERG channel protein was analyzed by Western blot. HEK293 cells expressing WT-hERG, E637KhERG and WT/E637K-hERG were incubated in PD-118057 or thapsigargin for 24 to 48 hours. As fig. 7A shows, HEK293 cells expressing WT-hERG undergo core and complex glycosylation during normal biogenesis, and the presence of two single protein bands at approximately $135 \mathrm{kDa}$ and $155 \mathrm{kDa}$ represents the hERG signature. The $155 \mathrm{kDa}$ band represents the complexly glycosylated (mature) form while the lower band $(135 \mathrm{kDa})$ represents the core-glycosylated (immature) form of the channel protein, which is retained in the ER $[17,19]$. The appearance of a $155 \mathrm{kDa}$ band is often used as an indicator of channel rescue [6,7,22]. E637K-hERG cells have an atypical hERG expression pattern with only a single band at $135 \mathrm{kDa}$. Moreover, cells expressing WT/E637K-hERG express two protein bands at approximately $135 \mathrm{kDa}$ and $155 \mathrm{kDa}$, but the $155 \mathrm{kDa}$ protein band is fainter than that of cells expressing WT-hERG. Fig. 7B-G show the Western blot analysis of cells expressing WT-hERG, E637K-hERG and WT/E637K-hERG protein channels before and after treatment with PD-118057 or thapsigargin. As expected with normal protein trafficking, WT-hERG expressing cells have both the $155 \mathrm{kDa}$ and $135 \mathrm{kDa}$ band, and PD-118057 and thapsigargin treatment had no effect on them at either 24 or 48 
hours. In cells expressing E637K-hERG, treatment with either drug also did not result in the appearance of a $155 \mathrm{kDa}$ band, consistent with failure to rescue. Similarly, cells expressing WT/ E637K-hERG were unaffected by PD-118057 or thapsigargin treatment. These data suggest that neither PD-118057 nor thapsigargin can rescue the dominant- negative effect of E637KhERG channel function.

\section{Discussion}

To date, treatment of LQT2 is inadequate. Beta-blockers are reported to be only $59 \%$ effective in preventing cardiac events in patients with LQT2 [23,24]. It has been suggested that drugs which activate cardiac $\mathrm{K}^{+}$channels could be used to enhance net repolarizing currents, which are reduced by gene mutations as well as hERG channel blockade. HERG channel activators prevent arrhythmia by suppressing action potential duration alternans $[25,26]$. PD-1 18057 is a novel hERG channel activator that does not cause hERG blockade, and is a potential therapeutic option for LQT2. Additionally, thapsigargin, an inhibitor of endoplasmic reticulum $\mathrm{Ca}^{2+}$-ATPase, has been shown to rescue LQT2associated KCNH2 mutations without hERG channel blockade. Our study investigates the effect of PD-118057 and thapsigargin on the current amplitude and gating properties of hERG and WT/E637K-hERG channels, and whether drug treatment can rescue channel processing and trafficking defects of the E637KhERG mutant, which has not been previously reported.

In our study, PD-118057 was shown to significantly enhance both the maximum current amplitude and tail current amplitude of WT-hERG cells. However, PD-118057 did not significantly alter the gating and kinetic properties of the WT-hERG channel, with the exception of accelerating steady-state inactivation. These results are consistent with a previous study which reported the activity of PD-118057 was voltage-dependent, enhanced the magnitude of hERG current, and did not significantly alter the gating and kinetic properties of the hERG channel [27]. In contrast to PD-118057, RPR260243 is a type I hERG agonist which enhances current magnitude by attenuating inactivation, as well as slowing hERG channel deactivation. Because the binding site of PD-118057 is a distant hydrophobic pocket formed by residues located on the pore helix, which is a nearby region of S6, PD-1 18057 does not directly affect deactivation [28]. Casis and colleagues [29] suggest that the decrease in rectification of hERG by PD-1 18057 indicates that it may have a similar mechanism as NS1643, which is another type 2 hERG agonist. NS1643 reduces the rectification of hERG through fast channel inactivation, thus slowing the onset of hERG inactivation. It is reported that a number of modulators positively regulate hERG channels, including phosphatidylinositol 4, 5-bisphosphate [30], PKC [31], direct binding of cAMP [32] and Polyamines [33]. However, their regulatory mechanisms often lack specificity, resulting in the functional alteration of other ion channels, and are typically associated with gating and/or kinetic changes. Although recent studies cannot rule out the possibility that PD-1 18057 acts through indirect mechanisms to increase hERG currents, our results showing the failure of PD-118057 to significantly affect the gating and kinetic properties of the hERG channel cause us to speculate that PD-118057 increases current amplitude by binding to the channel directly and increasing its open probability and activation potential.

Additionally, our study is the first to demonstrate that PD1 18057 is unable to rescue the dominant-negative suppression of the E637K-hERG mutant on WT-hERG channel function. This may be due to PD-118057 being a specific $\mathrm{I}_{\mathrm{Kr}}$ agonist, and having no effect on $\mathrm{I}_{\mathrm{Na}}, \mathrm{I}_{\mathrm{Ca}}, \mathrm{I}_{\mathrm{K} 1}$, or $\mathrm{I}_{\mathrm{Ks}}$ [27]. Given the requirement for $\mathrm{PD}-118057$ to bind directly to the channel, the reduction of open $\mathrm{I}_{\mathrm{Kr}}$ channel conformation seen with WT/ E637K-hERG may prevent PD-1 18057 from rescuing its function. Future work might be performed to test this hypothesis. We also found that PD-118057 failed to enhance the current magnitude of E637K-hERG channel. Given the hypothesis that PD-118057 binds to a distant hydrophobic pocket formed by residues located on the pore helix and a nearby region of S6, mutation in this region may abolish current activation by PD-118057 [34]. Further studies might be performed to test this hypothesis, such as using PD-1 18057 to treat several different missense mutations located on the pore helix or nearby region of S6, allowing us to assess the specific binding site of PD-118057.

Our study also demonstrates that thapsigargin significantly enhances both the maximum current amplitude and tail current amplitude of WT-hERG cells. For cell expressing the WT-hERG channel, thapsigargin treatment increased the maximal current amplitudes by $57.43 \%$ compared to control cells. Similarly, the tail current amplitude was increased $63.39 \%$ after thapsigargin treatment. However, thapsigargin did not significantly alter the gating or kinetic properties of the WT-hERG channel, with the exception of attenuating steady-state inactivation. Moreover, our study is the first to demonstrate that thapsigargin fails to rescue the dominant-negative suppression of the E637K-hERG mutant on WT-hERG channel function. It is possible that because WT/ E637K-hERG mutants consist of wild-type and E637K mutant channels, that such heteromultimers function as potassium channels but suppress normal channel function. Although thapsigargin significantly increased the WT-hERG channel current amplitude, it failed to rescue the E637K/WT-hERG current.

It is reported that dominant-negative mutation of hERG results in delayed myocellular repolarization and may promote L-type $\mathrm{Ca}^{2+}$ channel reactivation, leading to secondary depolarization and torsade de pointes arrhythmia [35-37]. Thapsigargin acts through inhibition of endoplasmic reticulum calcium ATPase, thus modulating the calcium-dependent chaperone proteins [13]. However, thapsigargin is unable to rescue all LQT2 mutations. It has been shown to rescue the G601S (S5/pore domain) and F805C (C-terminal junction) mutations, but not the N470D (S2 transmembrane domain) mutation [38-41]. Based on these reports, we hypothesized that thapsigargin targeted the region which is nearby the C-terminus. However, our study shows that thapsigargin fails to rescue trafficking defects of the E637K-hERG mutant, which is a novel missense mutation located in the pore-S6 loop transmembrane segment of hERG, nearby the C-terminus [14]. So in future work, we propose to investigate the molecular target for thapsigargin and whether thapsigargin can correct other LQT2 mutations.

Additionally, our Western blot results are consistent with the whole-cell patch-clamp recordings. When blotting for hERG protein, appearance of a $155-\mathrm{kDa}$ protein band is associated with normal hERG processing and is a marker for pharmacological rescue $[4,6,22]$. Our study shows that E637K-hERG cells expressed almost entirely as the $135 \mathrm{kDa}$ band after treatment with either PD-118057 or thapsigargin. Similarly, cells expressing WT/E637K-hERG were not rescued with either drug treatment. Taken together, our findings show that both PD-118057 and thapsigargin enhance WT-hERG channel current, but neither drug affects the current magnitude, gating kinetics or defective protein trafficking of the dominant negative WT/E637K-hERG mutant. Although not directly clinically applicable, these may impact the development of new therapies for diseases caused by 
the dominant-negative effect of LQT2 mutations. More importantly, experiments in animals or isolated hearts are needed to assess the potential therapeutic value and safety profile of PD118057 and thapsigargin.

\section{Supporting Information}

Table S1 Gandidate pharmacologic agents for the rescue of hERG current and their proposed mechanisms

\section{References}

1. Abbott GW, Sesti F, Splawski I, Buck ME, Lehmann MH, et al. (1999) MiRP1 forms IKr potassium channels with HERG and is associated with cardiac arrhythmia. Cell 97: 175-187.

2. Ficker E, Dennis AT, Obejero-Paz CA, Castaldo P, Taglialatela M, et al. (2000) Retention in the endoplasmic reticulum as a mechanism of dominant-negative current suppression in human long QT syndrome. J Mol Cell Cardiol 32: 23272337.

3. Ficker E, Thomas D, Viswanathan PC, Dennis AT, Priori SG, et al. (2000) Novel characteristics of a misprocessed mutant HERG channel linked to hereditary long QT syndrome. Am J Physiol Heart Circ Physiol 279: H17481756.

4. Furutani M, Trudeau MC, Hagiwara N, Seki A, Gong Q et al. (1999) Novel mechanism associated with an inherited cardiac arrhythmia: defective protein trafficking by the mutant HERG (G601S) potassium channel. Circulation 99: 2290-2294.

5. Zhou Z, Gong Q Epstein ML, January CT (1998) HERG channel dysfunction in human long QT syndrome. Intracellular transport and functional defects. J Biol Chem 273: 21061-21066.

6. Zhou Z, Gong Q January CT (1999) Correction of defective protein trafficking of a mutant HERG potassium channel in human long QT syndrome. Pharmacological and temperature effects. J Biol Chem 274: 31123-31126.

7. Ficker E, Obejero-Paz CA, Zhao S, Brown AM (2002) The binding site for channel blockers that rescue misprocessed human long QT syndrome type 2 ether-a-gogo-related gene (HERG) mutations. J Biol Chem 277: 4989-4998.

8. Lees-Miller JP, Duan Y, Teng GQ, Duff HJ (2000) Molecular determinant of high-affinity dofetilide binding to HERG1 expressed in Xenopus oocytes: involvement of S6 sites. Mol Pharmacol 57: 367-374.

9. Mitcheson JS, Chen J, Lin M, Culberson C, Sanguinetti MC (2000) A structural basis for drug-induced long QT syndrome. Proc Natl Acad Sci U S A 97: 12329-12333.

10. De Ponti F, Poluzzi E, Montanaro N (2000) QT-interval prolongation by noncardiac drugs: lessons to be learned from recent experience. Eur J Clin Pharmacol 56: 1-18.

11. De Ponti F, Poluzzi E, Montanaro N (2001) Organising evidence on QT prolongation and occurrence of Torsades de Pointes with non-antiarrhythmic drugs: a call for consensus. Eur J Clin Pharmacol 57: 185-209.

12. Sanguinetti MC, Mitcheson JS (2005) Predicting drug-hERG channel interactions that cause acquired long QT syndrome. Trends Pharmacol Sci 26: $119-124$.

13. Zhou Z, Gong Q, Ye B, Fan Z, Makielski JC, et al. (1998) Properties of HERG channels stably expressed in HEK 293 cells studied at physiological temperature. Biophys J 74: 230-241.

14. Hayashi K, Shimizu M, Ino H, Yamaguchi M, Mabuchi H, et al. (2002) Characterization of a novel missense mutation E637K in the pore-S6 loop of HERG in a patient with long QT syndrome. Cardiovasc Res 54: 67-76.

15. Sanguinetti MC, Tristani-Firouzi M (2006) hERG potassium channels and cardiac arrhythmia. Nature 440: 463-469.

16. Delisle BP, Anderson CL, Balijepalli RC, Anson BD, Kamp TJ, et al. (2003) Thapsigargin selectively rescues the trafficking defective LQT2 channels G601S and F805C. J Biol Chem 278: 35749-35754.

17. McPate MJ, Duncan RS, Milnes JT, Witchel HJ, Hancox JC (2005) The N588K-HERG K+ channel mutation in the 'short QT syndrome': mechanism of gain-in-function determined at 37 degrees C. Biochem Biophys Res Commun 334: 441-449.

18. Anson BD, Ackerman MJ, Tester DJ, Will ML, Delisle BP, et al. (2004) Molecular and functional characterization of common polymorphisms in HERG (KCNH2) potassium channels. Am J Physiol Heart Circ Physiol 286: H2434 2441.

19. Anderson CL, Delisle BP, Anson BD, Kilby JA, Will ML, et al. (2006) Most LQT2 mutations reduce Kv11.1 (hERG) current by a class 2 (traffickingdeficient) mechanism. Circulation 113: 365-373. of action. High variability exists in both the potential binding sites and resultant bioelectric alterations.

(DOG)

\section{Author Contributions}

Conceived and designed the experiments: NSL JFL. Performed the experiments: HYM XLL XYH XY YNB YW. Analyzed the data: JMK JQZ JFL. Contributed reagents/materials/analysis tools: HYM XYH YX JFL. Wrote the paper: HYM JMK.

20. Lian J, Huang N, Zhou J, Ge S, Huang X, et al. (2010) Novel characteristics of a trafficking-defective G572R-hERG channel linked to hereditary long QT syndrome. Can J Cardiol 26: 417-422.

21. Huang N, Lian JF, Huo JH, Liu LY, Ni L, et al. (2011) The EGFP/hERG fusion protein alter the electrophysiological properties of hERG channels in HEK293 cells. Cell Biol Int 35: 193-199.

22. Paulussen A, Raes A, Matthijs G, Snyders DJ, Cohen N, et al. (2002) A novel mutation (T65P) in the PAS domain of the human potassium channel HERG results in the long QT syndrome by trafficking deficiency. J Biol Chem 277: $48610-48616$.

23. Schwartz PJ, Priori SG, Spazzolini C, Moss AJ, Vincent GM, et al. (2001) Genotype-phenotype correlation in the long-QT syndrome: gene-specific triggers for life-threatening arrhythmias. Circulation 103: 89-95.

24. Priori SG, Napolitano C, Schwartz PJ, Grillo M, Bloise R, et al. (2004) Association of long QT syndrome loci and cardiac events among patients treated with beta-blockers. JAMA 292: 1341-1344.

25. Hua F, Gilmour RF, Jr. (2004) Contribution of IKr to rate-dependent action potential dynamics in canine endocardium. Circ Res 94: 810-819.

26. Hua F, Johns DC, Gilmour RF, Jr. (2004) Suppression of electrical alternans by overexpression of HERG in canine ventricular myocytes. Am J Physiol Heart Circ Physiol 286: H2342-2351.

27. Zhou J, Augelli-Szafran CE, Bradley JA, Chen X, Koci BJ, et al. (2005) Novel potent human ether-a-go-go-related gene ( $\mathrm{hERG}$ ) potassium channel enhancers and their in vitro antiarrhythmic activity. Mol Pharmacol 68: 876-884.

28. Kang J, Chen XL, Wang H, Ji J, Cheng H, et al. (2005) Discovery of a small molecule activator of the human ether-a-go-go-related gene (HERG) cardiac K+ channel. Mol Pharmacol 67: 827-836.

29. Casis O, Olesen SP, Sanguinetti MC (2006) Mechanism of action of a novel human ether-a-go-go-related gene channel activator. Mol Pharmacol 69: 658665.

30. Bian J, Cui J, McDonald TV (2001) HERG K(+) channel activity is regulated by changes in phosphatidyl inositol 4,5-bisphosphate. Circ Res 89: 1168-1176.

31. Thomas D, Zhang W, Wu K, Wimmer AB, Gut B, et al. (2003) Regulation of HERG potassium channel activation by protein kinase $\mathrm{C}$ independent of direct phosphorylation of the channel protein. Cardiovasc Res 59: 14-26.

32. Cui J, Melman Y, Palma E, Fishman GI, McDonald TV (2000) Cyclic AMP regulates the HERG $\mathrm{K}(+)$ channel by dual pathways. Curr Biol 10: 671-674.

33. Nichols CG, Lopatin AN (1997) Inward rectifier potassium channels. Annu Rev Physiol 59: 171-191.

34. Perry M, Sachse FB, Abbruzzese J, Sanguinetti MC (2009) PD-118057 contacts the pore helix of hERG1 channels to attenuate inactivation and enhance K+ conductance. Proc Natl Acad Sci U S A 106: 20075-20080.

35. January CT, Riddle JM (1989) Early afterdepolarizations: mechanism of induction and block. A role for L-type Ca2+ current. Circ Res 64: 977-990.

36. Surawicz B (1989) Electrophysiologic substrate of torsade de pointes: dispersion of repolarization or early afterdepolarizations? J Am Coll Cardiol 14: 172-184.

37. Antzelevitch C, Sicouri S (1994) Clinical relevance of cardiac arrhythmias generated by afterdepolarizations. Role of $\mathrm{M}$ cells in the generation of $\mathrm{U}$ waves, triggered activity and torsade de pointes. J Am Coll Cardiol 23: 259-277.

38. Akimoto K, Furutani M, Imamura S, Furutani Y, Kasanuki H, et al. (1998) Novel missense mutation (G601S) of HERG in a Japanese long QT syndrome family. Hum Mutat Suppl 1: S184-186.

39. Splawski I, Shen J, Timothy KW, Lehmann MH, Priori S, et al. (2000) Spectrum of mutations in long-QT syndrome genes. KVLQT1, HERG, SCN5A, KCNE1, and KCNE2. Circulation 102: 1178-1185.

40. Akhavan A, Atanasiu R, Noguchi T, Han W, Holder N, et al. (2005) Identification of the cyclic-nucleotide-binding domain as a conserved determinant of ion-channel cell-surface localization. J Cell Sci 118: 2803-2812.

41. Curran ME, Splawski I, Timothy KW, Vincent GM, Green ED, et al. (1995) A molecular basis for cardiac arrhythmia: HERG mutations cause long QT syndrome. Cell 80: 795-803. 